\title{
Angiotensin II in ECMO patients: a word of caution
}

\author{
Elio Antonucci ${ }^{1 *}$ (D) and Fabio Silvio Taccone ${ }^{2}$ \\ This comment refers to the article available at https://doi.org/10.1186/s13054-018-2225-4
}

We read with interest the recent letter by Ostermann et al. [1] about the administration of angiotensin II (Ang II) in patients treated by extracorporeal membrane oxygenation (ECMO). The authors reported seven patients with severe cardiorespiratory or respiratory failure receiving Ang II infusion during ECMO in the setting of high catecholamine doses. After Ang II initiation, they found a significant increase in blood pressure and a quick reduction in the need of other vasopressors. Despite the novelty of these findings, a word of caution should also be considered.

First, two patients were treated with veno-venous (VV) ECMO, that is often initiated in the case of severe acute respiratory distress syndrome (ARDS). Renin-angiotensin system (RAS) has been implicated in the pathogenesis of ARDS. In particular, Ang II is a pro-inflammatory molecule able to promote capillary leak and fibroproliferation. Also, Ang II may contribute to a significant constriction of the pulmonary circulation, potentially leading to reduced blood flow and to increased ventilation/perfusion mismatch [2]. Opposite to the angiotensin-converting enzyme (ACE)/Ang II axis, RAS has a counter-regulatory axis, which is composed by angiotensin-(1-7) (Ang 1-7) and ACE2 [2]. Since ACE2 can minimize the risk of acute lung injury, a recombinant form of human ACE2 (rhACE2) has been tested in patients with ARDS in a recent phase II trial [2]; this study showed that rhACE2 increases surfactant protein D concentrations without major side effects. Also, in an experimental model of ARDS, Ang 1-7 led to a significant improvement in oxygenation, decreasing the severity of acute lung injury and inflammation [3].

Second, five patients were treated with veno-arterial (VA) ECMO, that is usually implemented in cases of severe cardiac failure. No information on cardiac function was provided in this series, while the safety profile of Ang II has never been tested in patients with vasodilatory shock and severe concomitant myocardial dysfunction. Indeed, Ang II could reduce the cardiac output due to its vasoconstrictive properties and provide some detrimental effects on myocardial recovery [4].

Finally, ECMO patients need an adequate accurate anti-coagulation for the integrity of the extracorporeal system. Ang II usually exerts pro-coagulant effects, stimulating thrombin generation and potentially damaging microcirculation [5]. For all these reasons, we think that more safety and physiological data on the effects of Ang II should be collected before to extend its use in ECMO patients.

\section{Authors' response}

Marlies Ostermann and Kyle Gunnerson

We appreciate the comments of Professors Antunucci and Taccone who acknowledge the hemodynamic improvement associated with the use of angiotensin II (Ang II) in patients on ECMO requiring high-dose vasopressors but offer a word of caution related to ARDS and cardiogenic shock.

\footnotetext{
* Correspondence: elio.antonucci@yahoo.it

${ }^{1}$ Intermediate Care Unit - Emergency Department - Ospedale Guglielmo da

Saliceto, Piacenza, Italy

Full list of author information is available at the end of the article
}

With regard to ARDS, animal research has suggested that Ang II is detrimental in ARDS, hence the clinical development of recombinant ACE-2 (rhACE-2), an enzyme that converts Ang II into Ang 1-7. Studies in animals were promising. However, the clinical trial by Khan et al which Professors Antunucci and Taccone refer to, tested rhACE-2 in patients with ARDS and failed to show an improvement in oxygenation or any physiological parameters [2]. In fact, the trial was terminated 
early following a futility analysis. Interestingly, this study also showed that $71 \%$ of patients had low baseline concentrations of Ang II $<50 \mathrm{pg} / \mathrm{ml}$. This is in keeping with the results by Orfanos et al who demonstrated that severe ARDS was associated with angiotensin converting enzyme dysfunction resulting in Ang II deficiency [6]. Lastly, we would argue that a theoretical improvement in oxygenation, as suggested by animal studies, is less relevant during ECMO when oxygenation is provided via the extracorporeal circuit.

The authors' second point is that Ang II may decrease cardiac output. Whilst this is in theory possible, previous studies have shown that administration of bovine angiotensin II successfully rescued patients with cardiogenic shock, including patients in a cardiac arrest situation [7]. We would also like to point out that Ang I is largely converted to Ang II in the lungs. During VA ECMO, a significant proportion of blood is drawn via the venous cannula into the ECMO circuit and bypasses the lung. This is likely to contribute to Ang II deficiency and supports the case for Ang II in this setting [1].

Finally, Ang 1-7 is a potent vasodilator with effects opposite those of Ang II. Ang 1-7 builds up when ACE is not functional. The administration of Ang II may decrease Ang 1-7 via biofeedback resulting in an increase of blood pressure and decrease of the catecholamine dose [8].

We fully agree that more research is needed to determine the role of Ang II in patients on ECMO. Given that the bovine form of angiotensin II was utilized safely and effectively for over 45 years to treat shock, data in the literature may help us developing thoughtful prospective trials.

\footnotetext{
Abbreviations

ACE: Angiotensin-converting enzyme; Ang 1-7: Angiotensin 1-7; Ang II: Angiotensin II; ARDS: Acute respiratory distress syndrome; ECMO: Extracorporeal membrane oxygenation; RAS: Renin-angiotensin system; rhACE2: Recombinant form of human ACE2; VA: Veno-arterial; W: Veno-venous
}

Funding

N/A

\section{Availability of data and materials}

N/A

\section{Authors' contributions}

EA and FST wrote the article. Both authors read and approved the final manuscript.

\section{Ethics approval and consent to participate}

N/A

Consent for publication

N/A

\section{Competing interests}

The authors declare that they have no competing interests.

\section{Publisher's Note}

Springer Nature remains neutral with regard to jurisdictional claims in published maps and institutional affiliations.

\section{Author details}

${ }^{1}$ Intermediate Care Unit - Emergency Department - Ospedale Guglielmo da Saliceto, Piacenza, Italy. ${ }^{2}$ Department of Intensive Care, Laboratoire de

Recherche Experimentale, Erasme Hospital, Brussels, Belgium.

Received: 26 December 2018 Accepted: 28 January 2019

Published online: 26 April 2019

References

1. Ostermann M, Boldt DW, Harper MD, Lim GW, Gunnerson K. Angiotensin in ECMO patients with refractory shock. Crit Care. 2018;22(1):288.

2. Khan A, Benthin C, Zeno B, Albertson TE, Boyd J, Christie JD. A pilot clinical trial of recombinant human angiotensin-converting enzyme 2 in acute respiratory distress syndrome. Crit Care. 2017;21(1):234.

3. Zambelli V, Bellani G, Borsa R, Pozzi F, Grassi A, Scanziani M, et al. Angiotensin-(1-7) improves oxygenation, while reducing cellular infiltrate and fibrosis in experimental acute respiratory distress syndrome. Intensive Care Med Exp. 2015;3:44.

4. Antonucci E, Agosta S, Sakr Y. Angiotensin II in vasodilatory shock: lights and shadows. Crit Care. 2017;21:277.

5. Senchenkova EY, Russell J, Vital SA, Yildirim A, Orr AW, Granger DN. A critical role for both CD40 and VLA5 in angiotensin II-mediated thrombosis and inflammation. FASEB J. 2018;32:3448-56.

6. Orfanos SE, Armaganidis A, Glynos C et al. Pulmonary capillary endotheliumbound angiotensin-converting enzyme activity in acute lung injury. Circulation. 2000;102(16):2011-8.

7. Busse LW, McCurdy MT, Ali O, Hall A, Chen H, Ostermann M. The effect of angiotensin II on blood pressure in patients with circulatory shock: a structured review of the literature. Crit Care. 2017;21(1):324.

8. Chawla LS, Chen S, Bellomo R, Tidmarsh GF. Angiotensin converting enzyme defects in shock: implications for future therapy. Crit Care 2018; 22(1):274. 